\title{
On Toda's Theorem in structural communication complexity
}

Henning Wunderlich

\section{Ulmer Informatik-Berichte}

Nr. 2008-12

September 2008 



\title{
On Toda's Theorem in structural communication complexity
}

\author{
Henning Wunderlich *
}

August 27, 2008

\begin{abstract}
We prove Toda's Theorem in the context of structural communication complexity, i.e. $\mathbf{P} \mathbf{H}^{c c} \subseteq \mathrm{BP} \cdot \oplus \mathbf{P}^{c c} \subseteq \mathbf{P}^{c c}\left(\# \mathbf{P}^{c c}\right)=\mathbf{P}^{c c}\left(\mathbf{P P}^{c c}\right)$. The class PSPACE $^{c c}$ was defined via alternating protocols with $\mathcal{O}(\log n)$ many alternations. We consider the class BP $\oplus \oplus \mathbf{P}^{c c}$ of Toda's Theorem, and show that every language in this class can be decided with alternating protocols using $\mathcal{O}(\log n / \log \log n)$ many alternations. The proof is based on a new alternating protocol for the inner product function IP with $\mathcal{O}(\log n / \log \log n)$ many alternations.
\end{abstract}

\section{Introduction}

The main contribution of this paper is to establish Toda's Theorem in the setting of communication complexity, i.e. we prove $\mathbf{P} \mathbf{H}^{c c} \subseteq \mathrm{BP} \cdot \oplus \mathbf{P}^{c c} \subseteq \mathbf{P}^{c c}\left(\# \mathbf{P}^{c c}\right)=$ $\mathbf{P}^{c c}\left(\mathbf{P} \mathbf{P}^{c c}\right)$. This might be useful in the search for a solution of the famous $\mathbf{P} \mathbf{H}^{c c}$ vs. PSPACE ${ }^{c c}$ problem, because no communication complexity measures/lower bound methods are known for alternating classes, while for the classes $\mathbf{B P P} \mathbf{P}^{c c}$ and $\oplus \mathbf{P}^{c c}$ lower bound methods are available. Thus, it might be easier to come up with a measure for $\mathrm{BP} \cdot \oplus \mathbf{P}^{c c}$, a class not based on the concept of alternation, than to develop a measure for alternation. Of course, it might be the case that $\mathrm{BP} \cdot \oplus \mathbf{P}^{c c}=\mathbf{P S P A C E}^{c c}$, but we show that every language in $\mathrm{BP} \cdot \oplus \mathbf{P}^{c c}$ can be decided with alternating protocols using only $\mathcal{O}(\log n / \log \log n)$ many alternations, i.e. substantially less than allowed for PSPACE $^{c c}$. The proof is based on a new alternating protocol for the inner product function IP with $\mathcal{O}(\log n / \log \log n)$ many alternations.

\subsection{Structural complexity}

For introductions to the broad field of structural complexity see $[3,2,6,14,10]$. Nice surveys on a variety of topics in this field can be found in [18, 19], especially on counting complexity in [15,7] by Schöning and Fortnow, respectively. The parity class $\oplus \mathbf{P}$ was defined by Papadimitriou and Zachos in [12], where it was shown that $\oplus \mathbf{P}(\oplus \mathbf{P})=\oplus \mathbf{P}$. One can define operators on complexity classes, e.g. the BP-operator, which was defined by Schöning [16]. Using the BP-operator

*Universität Ulm, Fakultät für Ingenieurwissenschaften und Informatik, Institut für Theoretische Informatik, Oberer Eselsberg, D-89069 Ulm, e-mail: Henning. Wunderlich@uni-ulm.de 
and the Valiant-Vazirani-Lemma [21] Toda [20] was able to prove his celebrated theorem

$$
\mathbf{P H} \subseteq \mathrm{BP} \cdot \oplus \mathbf{P} \subseteq \mathbf{P}(\# \mathbf{P})=\mathbf{P}(\mathbf{P P})
$$

which tells us that counting (mod 2 with random source) is at least as powerful as the whole polynomial-time hierarchy $\mathbf{P H}$. See also [17] for a simplified proof.

\subsection{Communication complexity}

For a thorough introduction to communication complexity we refer the reader to the book of Kushilevitz and Nisan [11].

\subsubsection{Basic definitions and notation.}

We only work with the binary alphabet $\mathbb{B}:=\{0,1\}$. The length of a string $x \in \mathbb{B}^{*}$ is denoted by $|x|$. A prefix-free encoding of $x$ is $\bar{x}:=0^{|x|} 1 x$. In order to encode pairs of strings $x, y \in \mathbb{B}^{*}$ we use the pairing function $\langle x, y\rangle:=\bar{x} y$. The set of pairs of strings of equal length is denoted by $\mathbb{B}^{* *}:=\left\{(x, y)\left|x, y \in \mathbb{B}^{*},\right| x|=| y \mid\right\}$. A language $L$ is a subset of $\mathbb{B}^{* *}$, its characteristic function $\chi^{L}$ is defined as $\chi^{L}:=\left(\chi_{n}^{L}\right)$, where $\chi_{n}^{L}: \mathbb{B}^{n} \times \mathbb{B}^{n} \rightarrow \mathbb{N}, \chi_{n}^{L}(x, y):=1$, if $(x, y) \in L$, and 0 otherwise. We write $(x, y) \in L$ if $\chi_{|x|}^{L}(x, y)=1$. The set of all languages is denoted by $\mathcal{L}$. A (communication) complexity class is a subset $\mathcal{C} \subseteq \mathcal{L}$. We define poly $:=\left\{f: \mathbb{R}^{+} \rightarrow \mathbb{R}^{+} \mid \exists\right.$ polynomial $\left.p: f \leq p\right\}$, the set of functions with polynomial growth. With log we denote the logarithm to the basis 2 .

\subsubsection{Yao's model.}

We consider the basic model of communication complexity, introduced by Yao [22]. In this model, there are two players (parties) Alice and Bob, who want to cooperatively compute a function $f: \mathcal{X} \times \mathcal{Y} \rightarrow \mathcal{Z}$, where $\mathcal{X}, \mathcal{Y}$ and $\mathcal{Z}$ are finite sets. Both have complete information about $f$ and unlimited computational power but receive only parts of the inputs. Alice is given $x \in \mathcal{X}$, Bob is given $y$, and they exchange messages in order to compute $f(x, y)$. Each message solely depends on the player's input and the messages communicated so far. The communication is carried out according to a fixed protocol $\Pi$ (over domain $\mathcal{X} \times \mathcal{Y}$ with range $\mathcal{Z})$.

\subsubsection{Protocols.}

There are four kinds of protocols, namely deterministic, randomized, nondeterministic and alternating ones. We only describe deterministic protocols in detail: A deterministic protocol is a labeled binary tree, where an inner node specifies the player who sends a bit of communication next. If $v$ is an inner node, then it is labeled either by a function $a_{v}: \mathcal{X} \rightarrow\{0,1\}$ or by a function $b_{v}: \mathcal{Y} \rightarrow\{0,1\}$. Each leaf $l$ is labeled with an output value $z_{l} \in \mathcal{Z}$. The value of the protocol $\Pi$ on input $(x, y)$ is the label of the leaf reached by starting from the root, and walking on the tree. At each internal node $v$ labeled by $a_{v}$ Alice sends $a_{v}(x)$ and they walk left if $a_{v}(x)=0$ and right if $a_{v}(x)=1$. Analogously, if $v$ is labeled with $b_{v}$. The cost of the protocol $\Pi$ on input $(x, y)$ is the length of the path taken on this input. In a randomized protocol Alice and Bob have access to a public or private source of randomness (random string). The functions $a_{v}, b_{v}$ are 
arbitrary functions of the inputs and the random strings. In a nondeterministic protocol, we have $\mathcal{Z}=\{0,1\}$, and each player gets a guess string in addition to the input. Here, $a_{v}$ and $b_{v}$ are arbitrary functions of the inputs and the guess strings. For nondeterministic protocols there exist different accepting modes. For example, a nondeterministic protocol accepts a language $L$ in the nondeterministic accepting mode, if for all $(x, y) \in L$ there exist guess strings $g_{A}$ and $g_{B}$ such that Alice on input $x$ and guess string $g_{A}$ and Bob on input $y$ and guess string $g_{B}$ reach a leaf labeled with 1 , and if for all $(x, y) \notin L$ there do not exist any guess strings such that the players reach a 1-leaf. Another example is the parity accepting mode: Here, an input is accepted iff the number of guess strings such that the players reach a 1-leaf is odd. For a definition of alternating protocols, see $[1$, p.339]. For formal definitions concerning protocols, cost and complexity measures, and accepting modes, see [11, Def. 1.1, p.4; Chap. 3, p.28; Chap. 2, p.18] and [5]. A protocol over domain $\mathcal{X} \times \mathcal{Y}$ is an $n$-bit protocol, if $\mathcal{X}=\mathcal{Y}=\mathbb{B}^{n}$. A protocol family $\left(\Pi_{n}\right)_{n \in \mathbb{N}}$ of $n$-bit protocols $\Pi_{n}$ decides a language $L$ if each $\Pi_{n}$ computes $\chi_{n}^{L}$.

\subsubsection{Communication complexity classes.}

Each protocol type and acceptance mode leads to a complexity measure, e.g. $\mathrm{D}(f)$ for the deterministic communication complexity of $f$, or $\oplus \mathrm{D}(f)$, which is the minimum cost of a nondeterministic protocol deciding $f$ in parity accepting mode. If a problem can be solved with communication polylogarithmically in the input size, then we consider this as efficient. The communication complexity classes are defined as sets of languages that can be decided efficiently according to a fixed measure. For example, $\mathbf{P}^{c c}$ is the class of languages such that $L \in \mathbf{P}^{c c}$ iff there exists a bound $b \in$ poly with $\mathrm{D}\left(\chi_{n}^{L}\right) \leq p(\log n)$, and $\oplus \mathbf{P}^{c c}$ is the class of languages such that $L \in \oplus \mathbf{P}^{c c}$ iff there exists a bound $b \in$ poly with $\oplus \mathrm{D}\left(\chi_{n}^{L}\right) \leq p(\log n)$.

\subsubsection{Oracle protocols.}

A deterministic, randomized, nondeterministic or alternating protocol $\Pi$ over $\mathcal{X}$, $\mathcal{Y}$ is an oracle protocol with oracle family $O=\left(O_{m}\right)_{m \in \mathbb{N}}$, if $\Pi$ contains oracle nodes in its protocol tree. Associated with an oracle node $v$ are two functions $a_{v}: \mathcal{X} \rightarrow \mathbb{B}^{m_{v}}$ and $b_{v}: \mathcal{Y} \rightarrow \mathbb{B}^{m_{v}}$. If Alice and Bob reach an oracle node $v$ during a computation on input $(x, y) \in X \times \mathcal{Y}$, they compute by themselves $x^{\prime}:=a_{v}(x)$ and $y^{\prime}:=b_{v}(y)$, respectively, and call $O_{m_{v}}$ on $\left(x^{\prime}, y^{\prime}\right)$. The oracle node $v$ has exactly $\mid$ range $(O) \mid$ many successors. Alice and Bob continue the computation on one of them according to the returned value $O\left(x^{\prime}, y^{\prime}\right)$. The communication costs for each oracle call are $\lceil\log |\operatorname{range}(O)|\rceil$. If a language $L$ is used as an oracle family, we write $L$ instead of $\chi^{L}$. Relativized communication complexity classes are defined via efficient oracle protocol families. For example, $\mathbf{P}^{c c}\left(L^{\prime}\right)$ contains all languages $L$ which can be decided by a protocol family $\left(\Pi_{n}\right)_{n \in \mathbb{N}}$ of deterministic $n$-bit oracle protocols with $L^{\prime}$ as the oracle.

\subsection{Structural communication complexity}

Research in the field of structural communication complexity started with the article of Babai, Frankl and Simon [1], where some analogies between Turing ma- 
chine classes like $\mathbf{P}, \mathbf{N P}, \mathbf{P P}, \mathbf{P S P A C E}$, the polynomial hierachy $\mathbf{P H}=\bigcup_{k} \Sigma_{k}^{p}$, etc. and the corresponding communication complexity classes $\mathbf{P}^{c c}, \mathbf{N} \mathbf{P}^{c c}, \mathbf{P P}^{c c}$, PSPACE ${ }^{c c}, \mathbf{P H}{ }^{c c}=\bigcup_{k} \Sigma_{k}^{c c}$, etc. were shown. For more ground work, especially on closure properties, the boolean communication hierarchy, or counting communication complexity classes like $\mathrm{MOD}_{m} \mathbf{P}$, see Halstenberg and Reischuk [8] or Damm et al. [5]. In [9] Klauck established separation results between $\mathbf{M A}^{c c}$ and $\mathbf{N P} \mathbf{P}^{c c}, \mathbf{M} \mathbf{A}^{c c}$ and $\mathbf{A} \mathbf{P} \mathbf{P}^{c c}$, and $\mathbf{A} \mathbf{P} \mathbf{P}^{c c}$ and $\mathbf{P} \mathbf{P}^{c c}$, respectively. In recent research, Buhrman et al. [4] showed $\Sigma_{2}^{c c}, \Pi_{2}^{c c} \nsubseteq \mathbf{P P}^{c c}$. This was improved to $\Sigma_{2}^{c c}, \Pi_{2}^{c c} \nsubseteq \mathbf{U P P}^{c c}$ by Razborov and Sherstov [13].

\subsubsection{Reductions.}

We introduce different kinds of reductions between languages. The many-one reductions are also called rectangular reductions. The disjunctive reductions are not needed in the sequel but defined only for the sake of completeness.

Definition 1.1. (Reductions) Let $L$ and $L^{\prime}$ be languages.

1. $L$ is many-one reducible to $L^{\prime}$, if there exists $a$ bound $b \in$ poly and $a$ family of function pairs $\left\{\left(f_{n}, g_{n}\right)\right\}_{n \in \mathbb{N}}, f_{n}, g_{n}: \mathbb{B}^{n} \rightarrow \mathbb{B}^{\left\lceil 2^{b(\log n)}\right\rceil}$, such that for all $(x, y) \in\left(\mathbb{B}^{n}\right)^{2}$ it holds: $(x, y) \in L$ iff $\left(f_{n}(x), g_{n}(y)\right) \in L^{\prime}$.

2. $L$ is Turing reducible to $L^{\prime}$, if $L \in \mathbf{P}^{c c}\left(L^{\prime}\right)$.

3. $L$ is majority reducible to $L^{\prime}$, if there exist bounds $b, t \in$ poly and a family of function pairs $\left\{\left(f_{n}, g_{n}\right)\right\}_{n \in \mathbb{N}}, f_{n}, g_{n}: \mathbb{B}^{n} \rightarrow\left(\mathbb{B}^{\left\lceil 2^{b(\log n)}\right\rceil}\right)^{\lceil t(\log n)\rceil}$, such that for all $(x, y) \in\left(\mathbb{B}^{n}\right)^{2}$ it holds: $(x, y) \in L$ iff $\left(\left(f_{n}(x)\right)_{i},\left(g_{n}(y)\right)_{i}\right) \in L^{\prime}$ for the majority of the indices $i \in[\lceil t(\log n)\rceil]$.

4. $L$ is conjunctively reducible to $L^{\prime}$, if there exist bounds $b, t \in$ poly and a family of function pairs $\left\{\left(f_{n}, g_{n}\right)\right\}_{n \in \mathbb{N}}, f_{n}, g_{n}: \mathbb{B}^{n} \rightarrow\left(\mathbb{B}^{\left\lceil 2^{b(\log n)}\right\rceil}\right)^{\lceil t(\log n)\rceil}$, such that for all $(x, y) \in\left(\mathbb{B}^{n}\right)^{2}$ it holds: $(x, y) \in L$ iff $\left(\left(f_{n}(x)\right)_{i},\left(g_{n}(y)\right)_{i}\right) \in$ $L^{\prime}$ for all indices $i \in[\lceil t(\log n)\rceil]$.

5. $L$ is disjunctively reducible to $L^{\prime}$, if there exist bounds $b, t \in$ poly and $a$ family of function pairs $\left\{\left(f_{n}, g_{n}\right)\right\}_{n \in \mathbb{N}}, f_{n}, g_{n}: \mathbb{B}^{n} \rightarrow\left(\mathbb{B}^{\left\lceil 2^{b(\log n)}\right\rceil}\right)^{\lceil t(\log n)\rceil}$, such that for all $(x, y) \in\left(\mathbb{B}^{n}\right)^{2}$ it holds: $(x, y) \in L$ iff $\left(\left(f_{n}(x)\right)_{i},\left(g_{n}(y)\right)_{i}\right) \in$ $L^{\prime}$ for at least one of the indices $i \in[\lceil t(\log n)\rceil]$.

\subsection{Organization of this paper}

In Section 1 we prove Toda's Theorem in the setting of communication complexity. In Section 2 we present an alternating protocol for the inner product function IP with $\mathcal{O}(\log n / \log \log n)$ many alternations, which gives us an upper bound on the number of alternations for languages in the class $\mathrm{BP} \cdot \oplus \mathbf{P}^{c c}$ of Toda's Theorem.

\section{Toda's Theorem}

In order to prove Toda's Theorem we need to define different kinds of operators on communication complexity classes. Readers familiar with communication 
complexity might wonder why the operators are defined in a public coin style, i.e. both players get the same witness/random string. Of course, one can define the operators such that each player gets his/her own witness/random string (private coin style). The reason is that these definitions are equivalent, if the operators are simulated by a protocol. Alice can guess Bob's witness and send it to him, or she can send him her random string, because the length of witnesses/random strings is bounded polylogarithmically in the length of the input.

Definition 2.1. (Complexity class operators) For a language $L \subseteq \mathbb{B}^{* *}$ and bounds $p, q \in$ poly we define

$$
\begin{aligned}
\forall^{p}(L) & :=\left\{(x, y) \in \mathbb{B}^{* *} \mid \forall w \in \mathbb{B}^{\lceil p(\log |x|)\rceil}:(\langle x, w\rangle,\langle y, w\rangle) \in L\right\}, \\
\exists^{p}(L) & :=\left\{(x, y) \in \mathbb{B}^{* *} \mid \exists w \in \mathbb{B}^{\lceil p(\log |x|)\rceil}:(\langle x, w\rangle,\langle y, w\rangle) \in L\right\}, \\
\operatorname{Mod}_{k}^{p}(L) & :=\left\{(x, y) \in \mathbb{B}^{* *}||\left\{w \in \mathbb{B}^{\lceil p(\log |x|)\rceil} \mid(\langle x, w\rangle,\langle y, w\rangle) \in L\right\} \mid \bmod k \neq 0\right\}, \\
\oplus^{p}(L) & :=\operatorname{Mod}_{2}^{p}(L) .
\end{aligned}
$$

For a communication complexity class $\mathcal{C} \subseteq \mathcal{L}$ we define

$$
\begin{aligned}
\operatorname{co} \cdot \mathcal{C} & :=\{\bar{L} \mid L \in \mathcal{C}\}, \\
\forall \cdot \mathcal{C} & :=\left\{\forall^{p}(L) \mid L \in \mathcal{C}, p \in \text { poly }\right\} \\
\exists \cdot \mathcal{C} & :=\left\{\exists^{p}(L) \mid L \in \mathcal{C}, p \in \text { poly }\right\} \\
\operatorname{Mod}_{k} \cdot \mathcal{C} & :=\left\{\operatorname{Mod}_{k}^{p}(L) \mid L \in \mathcal{C}, p \in \text { poly }\right\} \\
\oplus \cdot \mathcal{C} & :=\operatorname{Mod}_{2} \cdot \mathcal{C} .
\end{aligned}
$$

A language $L$ is in $\mathrm{BP} \cdot \mathcal{C}$ if there exists a language $L^{\prime} \in \mathcal{C}$ and a bound $q \in$ poly such that for all $(x, y) \in\left(\mathbb{B}^{n}\right)^{2}$ it holds:

$$
\begin{aligned}
& (x, y) \in L \text { implies }\left|\left\{r \in \mathbb{B}^{\lceil q(\log n)\rceil} \mid(\langle x, r\rangle,\langle y, r\rangle) \in L^{\prime}\right\}\right| / 2^{\lceil q(\log n)\rceil} \geq \frac{2}{3} . \\
& (x, y) \notin L \text { implies }\left|\left\{r \in \mathbb{B}^{\lceil q(\log n)\rceil} \mid(\langle x, r\rangle,\langle y, r\rangle) \in L^{\prime}\right\}\right| / 2^{\lceil q(\log n)\rceil} \leq \frac{1}{3} .
\end{aligned}
$$

We give a definition of the polynomial hierarchy suitable for our purposes based on the class operators defined above. Note that this definition is equivalent to the one given in [1].

Definition 2.2. (Polynomial hierarchy) $\mathbf{P} \mathbf{H}^{c c}:=\bigcup_{k \geq 0} \Sigma_{k}^{c c}$, where $\Sigma_{0}^{c c}:=\mathbf{P}^{c c}$ and $\Sigma_{k+1}^{c c}:=\exists \cdot \mathrm{co} \cdot \Sigma_{k}^{c c}$.

We observe the following properties of the communication complexity class operators. The proofs are easy, so we omit most of them for space reasons.

Observation 2.3. (Probability amplification) Let $\mathcal{C} \subseteq \mathcal{L}$ be a communication complexity class closed under majority reductions, and let $b \in$ poly. If a language $L$ is in $\mathrm{BP} \cdot \mathcal{C}$, then there exists a language $L^{\prime} \in \mathcal{C}$ and a bound $q \in$ poly such that for all $(x, y) \in\left(\mathbb{B}^{n}\right)^{2}$ it holds:

$$
\begin{aligned}
& (x, y) \in L \Rightarrow\left|\left\{r \in \mathbb{B}^{\lceil q(\log n)\rceil} \mid(\langle x, r\rangle,\langle y, r\rangle) \in L^{\prime}\right\}\right| / 2^{\lceil q(\log n)\rceil} \geq 1-2^{-b(\log n)} . \\
& (x, y) \notin L \Rightarrow\left|\left\{r \in \mathbb{B}^{\lceil q(\log n)\rceil} \mid(\langle x, r\rangle,\langle y, r\rangle) \in L^{\prime}\right\}\right| / 2^{\lceil q(\log n)\rceil} \leq 2^{-b(\log n)} .
\end{aligned}
$$

Observation 2.4. (Inclusion) Let $\mathcal{C} \subseteq \mathcal{L}$ be a communication complexity class closed under many-one reductions. Then $\mathcal{C} \subseteq \mathrm{Op} \cdot \mathcal{C}$ for every operator $\mathrm{Op} \in$ $\left\{\forall, \exists, \operatorname{Mod}_{k}, \oplus, \mathrm{BP}\right\}$. 
Observation 2.5. (Monotonicity) Let $\mathcal{C}, \mathcal{D} \subseteq \mathcal{L}$ be communication complexity classes such that $\mathcal{C} \subseteq \mathcal{D}$. Then $\mathrm{Op} \cdot \mathcal{C} \subseteq \mathrm{Op} \cdot \mathcal{D}$ for every operator $\mathrm{Op} \in$ $\left\{\mathrm{co}, \forall, \exists, \operatorname{Mod}_{k}, \oplus, \mathrm{BP}\right\}$.

Observation 2.6. (Idempotency) Let $\mathcal{C} \subseteq \mathcal{L}$ be a communication complexity class closed under many-one reductions. Then $\mathrm{Op} \cdot \mathrm{Op} \cdot \mathcal{C}=\mathrm{Op} \cdot \mathcal{C}$ for every operator $\mathrm{Op} \in\{\forall, \exists, \oplus\}$.

The idempotency of the BP-operator follows from its probability amplification property (Observation 2.3).

Observation 2.7. (Idempotency of $B P$ ) Let $\mathcal{C} \subseteq \mathcal{L}$ be a communication complexity class closed under majority reductions. Then $\mathrm{BP} \cdot \mathrm{BP} \cdot \mathcal{C}=\mathrm{BP} \cdot \mathcal{C}$.

Observation 2.8. (co. vs. $\cdots)$ Let $\mathcal{C} \subseteq \mathcal{L}$ be a communication complexity class. Then co $\cdot \exists \cdot \mathcal{C}=\forall \cdot \operatorname{co} \cdot \mathcal{C}$, co $\forall \forall \cdot \mathcal{C}=\exists \cdot \mathrm{co} \cdot \mathcal{C}$, and co $\cdot \mathrm{BP} \cdot \mathcal{C}=\mathrm{BP} \cdot \operatorname{co} \cdot \mathcal{C}$.

Definition 2.9. (Intersection and Union) Let $\mathcal{C}$ and $\mathcal{C}^{\prime}$ be communication complexity classes. $\mathcal{C}$ is closed under $\mathcal{C}^{\prime}$-intersection iff for all $L \in \mathcal{C}$ and $L^{\prime} \in \mathcal{C}^{\prime}$ we have $L \cap L^{\prime} \in \mathcal{C} . \mathcal{C}$ is closed under $\mathcal{C}^{\prime}$-union iff for all $L \in \mathcal{C}$ and $L^{\prime} \in \mathcal{C}^{\prime}$ we have $L \cup L^{\prime} \in \mathcal{C}$.

Observation 2.10. (co-vs. $\oplus$ ) Let $\mathcal{C} \subseteq \mathcal{L}$ be a communication complexity class that contains $\mathbf{P}^{c c}$, is closed under $\mathbf{P}^{c c}$-intersection, $\mathbf{P}^{c c}$-union, and many-one reductions. Then $\mathrm{co} \cdot \oplus \cdot \mathcal{C}=\oplus \cdot \mathcal{C}$.

Proof. Let $L \in \oplus \cdot \mathcal{C}$. There exist a bound $p \in$ poly and a language $L_{1} \in \mathcal{C}$ such that $L=\oplus^{p}\left(L_{1}\right)$. Define

$$
\begin{aligned}
& L_{2}:=\left\{\left(\left\langle x, b_{1} w_{1}\right\rangle,\left\langle y, b_{2} w_{2}\right\rangle\right) \mid b_{1}, b_{2} \in \mathbb{B},\left(\left\langle x, w_{1}\right\rangle,\left\langle y, w_{2}\right\rangle\right) \in L_{1}\right\}, \\
& L_{3}:=\left\{\left(\left\langle x, 1 w_{1}\right\rangle,\left\langle y, 1 w_{2}\right\rangle\right)|| x|=| y|=n,| w_{1}|=| w_{2} \mid=\lceil p(\log n)\rceil\right\} \\
& L_{4}:=\left\{\left(\left\langle x, 0 w_{1}\right\rangle,\left\langle y, 0 w_{2}\right\rangle\right)|| x|=| y \mid=n, w_{1}=w_{2}=0^{\lceil p(\log n)\rceil}\right\} .
\end{aligned}
$$

Then $L_{2}$ is in $\mathcal{C}$, because $\mathcal{C}$ is closed under many-one reductions, and $L_{3}, L_{4} \in$ $\mathbf{P}^{c c}$. The language $L_{5}:=\left(L_{2} \cap L_{3}\right) \cup L_{4}$ is in $\mathcal{C}$, because $\mathcal{C}$ is closed under $\mathbf{P}^{c c_{-}}$ intersection and $\mathbf{P}^{c c}$-union. Define $L^{\prime}:=\oplus^{p+1}\left(L_{5}\right)$. Clearly, $\bar{L}=L^{\prime} \in \oplus \cdot \mathcal{C}$.

Observation 2.11. If $\mathcal{C} \subseteq \mathcal{L}$ is a communication complexity class closed under conjunctive reductions, then $\oplus \cdot \mathcal{C}$ is closed under conjunctive reductions.

Using Observations 2.10 and 2.11 one can prove the result of Papadimitriou and Zachos [12] in the setting of communication complexity as in time complexity (see also [10, Prop. 4.8, p.125]).

Fact 2.12. (Papadimitriou $\&$ Zachos) Let $\mathcal{C} \subseteq \mathcal{L}$ be a communication complexity class, which contains $\mathbf{P}^{c c}$, is closed under $\mathbf{P}^{c c}$-intersection, $\mathbf{P}^{c c}$-union, and conjunctive reductions. Then $\oplus \mathbf{P}^{c c}(\oplus \cdot \mathcal{C})=\oplus \cdot \mathcal{C}$.

The following observation shows that the names used for the operators are compatible with the names of classical communication complexity classes, if the operators are applied to $\mathbf{P}^{c c}$.

Observation 2.13. (Compatibility)

$$
\begin{aligned}
& \mathbf{N P}^{c c}=\exists \cdot \mathbf{P}^{c c}, \quad \oplus \mathbf{P}^{c c}=\oplus \cdot \mathbf{P}^{c c}, \\
& \mathbf{c o -} \mathbf{N} \mathbf{P}^{c c}=\forall \cdot \mathbf{P}^{c c}, \quad \mathbf{B P} \mathbf{P}^{c c}=\mathrm{BP} \cdot \mathbf{P}^{c c} \text {. }
\end{aligned}
$$


Swapping lemmata are well known in the field of structural complexity theory. Below, we give a proof of a lemma of this type for the sake of completeness. The main ingredient is the probability amplification property of the BP-operator (Observation 2.3).

Lemma 2.14. (Swapping) Let $\mathcal{C} \subseteq \mathcal{L}$ be a communication complexity class closed under majority reductions. Then $\oplus \cdot \mathrm{BP} \cdot \mathcal{C} \subseteq \mathrm{BP} \cdot \oplus \cdot \mathcal{C}$.

Proof. Let $L$ be a language in $\oplus \cdot \mathrm{BP} \cdot \mathcal{C}$. Then there exists a language $L^{\prime}$ in $\mathrm{BP} \cdot \mathcal{C}$ and a bound $p^{\prime} \in$ poly such that $L=\oplus^{p^{\prime}}\left(L^{\prime}\right)$. As $L^{\prime} \in \mathrm{BP} \cdot \mathcal{C}$ and $\mathcal{C}$ is closed under majority reductions we use probability amplification to obtain a language $L^{\prime \prime}$ in $\mathcal{C}$ and a bound $p^{\prime \prime} \in$ poly such that

$(\langle x, w\rangle,\langle y, w\rangle) \in L^{\prime} \Rightarrow \operatorname{Pr}_{r}\left[(\langle\langle x, w\rangle, r\rangle,\langle\langle y, w\rangle, r\rangle) \in L^{\prime \prime}\right] \geq 1-2^{-l_{n}^{\prime}-2}$, and $(\langle x, w\rangle,\langle y, w\rangle) \notin L^{\prime} \Rightarrow \operatorname{Pr}_{r}\left[(\langle\langle x, w\rangle, r\rangle,\langle\langle y, w\rangle, r\rangle) \in L^{\prime \prime}\right] \leq 2^{-l_{n}^{\prime}-2}$.

for every input $(x, y) \in\left(\mathbb{B}^{n}\right)^{2}$ and witness $w$. Here, $l_{n}^{\prime}:=\left\lceil p^{\prime}(\log n)\right\rceil$, and the random string $r$ is uniformly drawn from $\mathbb{B}^{l_{n}^{\prime \prime}}$, where $l_{n}^{\prime \prime}:=\left\lceil p^{\prime \prime}(\log n)\right\rceil$. We define $W_{(x, y)}:=\left\{w \in \mathbb{B}^{l_{n}^{\prime}} \mid(\langle x, w\rangle,\langle y, w\rangle) \in L^{\prime}\right\}$ and $\operatorname{Good}_{n}:=\bigcap_{w \in \mathbb{B}_{n}^{\prime \prime}} \operatorname{Good}_{n, w}$, where $\operatorname{Good}_{n, w}:=\left\{r \in \mathbb{B}_{n}^{l_{n}^{\prime \prime}} \mid \forall(x, y) \in\left(\mathbb{B}^{n}\right)^{2}:(\langle\langle x, w\rangle, r\rangle,\langle\langle y, w\rangle, r\rangle) \in L^{\prime \prime} \Leftrightarrow\right.$ $\left.w \in W_{(x, y)}\right\}$. For a fixed $w_{0}$ we get

$$
\operatorname{Pr}_{r}\left[r \notin \operatorname{Good}_{n}\right] \leq 2^{l_{n}^{\prime}} \cdot \operatorname{Pr}_{r}\left[r \notin \operatorname{Good}_{n, w_{0}}\right] \leq 2^{l_{n}^{\prime}} \cdot 2^{l_{n}^{\prime}-2} \leq \frac{1}{4} .
$$

Thus, $\operatorname{Pr}_{r}\left[r \in \operatorname{Good}_{n}\right] \geq \frac{3}{4}$. The language

$$
L^{\prime \prime \prime}:=\left\{\left(\langle\langle x, r\rangle, w\rangle,\left\langle\left\langle y, r^{\prime}\right\rangle, w^{\prime}\right\rangle\right) \mid\left(\langle\langle x, w\rangle, r\rangle,\left\langle\left\langle y, w^{\prime}\right\rangle, r^{\prime}\right\rangle\right) \in L^{\prime \prime}\right\}
$$

is in $\mathcal{C}$ (closure under many-one reductions).

In case $(x, y) \in L$ we have

$$
\begin{aligned}
& \operatorname{Pr}_{r}\left[(\langle x, r\rangle,\langle y, r\rangle) \in \oplus^{p^{\prime}}\left(L^{\prime \prime \prime}\right)\right] \\
= & \operatorname{Pr}_{r}\left[\left|\left\{w \mid(\langle\langle x, w\rangle, r\rangle,\langle\langle y, w\rangle, r\rangle) \in L^{\prime \prime}\right\}\right| \text { odd }\right] \\
\geq & \operatorname{Pr}_{r}\left[\forall w: w \in W_{(x, y)} \Leftrightarrow(\langle\langle x, w\rangle, r\rangle,\langle\langle y, w\rangle, r\rangle) \in L^{\prime \prime}\right] \\
\geq & \operatorname{Pr}_{r}\left[\forall(x, y): \forall w: w \in W_{(x, y)} \Leftrightarrow(\langle\langle x, w\rangle, r\rangle,\langle\langle y, w\rangle, r\rangle) \in L^{\prime \prime}\right] \\
= & \operatorname{Pr}_{r}\left[r \in \operatorname{Good}_{n}\right] \geq \frac{3}{4},
\end{aligned}
$$

where (1) follows from $(x, y) \in L \Leftrightarrow\left|W_{(x, y)}\right|$ odd.

The case $(x, y) \notin L$ is treated similarly. We conclude $L \in \mathrm{BP} \cdot \oplus \cdot \mathcal{C}$.

The Valiant-Vazirani-Lemma is well known in structural complexity theory, and there exist many proof ideas for this important result. The solution we propose is an adaptation of an algebraic proof due to Fortnow in [7, p.88, Lemma 3.12].

Lemma 2.15. (Valiant-Vazirani) Let $\mathcal{C} \subseteq \mathcal{L}$ be a communication complexity class containing $\mathbf{P}^{c c}$ and closed under $\mathbf{P}^{c c}$-intersection, $\mathbf{P}^{c c}$-union and conjunctive reductions. Then $\exists \cdot \mathcal{C} \subseteq \mathrm{BP} \cdot \oplus \cdot \mathcal{C}$.

Proof. Let $L$ be a language in $\exists \cdot \mathcal{C}$. There exists a language $L^{\prime} \in \mathcal{C}$ and a bound $p \in$ poly such that $L=\exists^{p}\left(L^{\prime}\right)$. Define $l_{n}:=\lceil p(\log n)\rceil$. We fix an input 
$(x, y) \in L,|x|=|y|=n$. Let $S:=\left\{w \in \mathbb{B}^{l_{n}} \mid(\langle x, w\rangle,\langle y, w\rangle) \in L^{\prime}\right\}$ be the set of witnesses of $(x, y)$ and $d:=|S|$ its size. We pick a natural number $m$ such that $2 l_{n} d<m \leq 4 l_{n} d$ and encode the witnesses as polynomials over $F:=$ $\mathrm{GF}\left(2^{m}\right)$, the finite field with $2^{m}$ elements. We then consider pairs $(a, b) \in F^{2}$ and show that for a sizable fraction of them there will be exactly one polynomial $p$ representing a witness such that $p(a)=b$. The statement follows by choosing $m, a$ and $b$ at random. For a string $s=s_{1} \cdots s_{l}$ we define the polynomial $p_{s}(X):=\sum_{i=1}^{l} s_{i} X^{i}$. We fix a witness $w$ in $S$. An element $a$ of $F$ is called $w$-good, if for all witnesses $w^{\prime} \neq w$ in $S$ we have $p_{w}(a) \neq p_{w^{\prime}}(a)$. Since $p_{w}$ and $p_{w^{\prime}}$ can agree on at most $l_{n}$ elements, there are at least $|F|-l_{n} d$ many $w$-good elements in $F$. Consider the set $A_{w}$ containing all pairs $\left(a, p_{w}(a)\right)$ for $w$-good elements $a$. The sets $A_{w}$ and $A_{w^{\prime}}$ are disjoint for different strings $w$ and $w^{\prime}$. Define $A:=\bigcup_{w \in S} A_{w}$. Then $|A| \geq d\left(|F|-l_{n} d\right)$. We define the language $L^{\prime \prime}$ in $\mathcal{C}$ by

$$
\begin{aligned}
L^{\prime \prime}:= & \left\{(\langle\langle x, r\rangle, w\rangle,\langle\langle y, r\rangle, w\rangle)|n:=| x|=| y \mid, r=\left\langle m^{*}, a, b\right\rangle, m^{*} \in\left[2 l_{n}\right],\right. \\
& \left.a, b \in \operatorname{GF}\left(2^{m^{*}}\right),|w|=l_{n}, p_{w}(a)=b,(\langle x, w\rangle,\langle y, w\rangle) \in L^{\prime}\right\},
\end{aligned}
$$

where $r=\left\langle m^{*}, a, b\right\rangle$ means that we use $r$ as an encoding of a natural number $m^{*}$ and field elements $a$ and $b$. Furthermore, define $L^{\prime \prime \prime}:=\oplus^{p}\left(L^{\prime \prime}\right) \in \oplus \cdot \mathcal{C}$.

If $(x, y) \notin L$ then for all $w$ and $r$ the pair $(\langle\langle x, r\rangle, w\rangle,\langle\langle y, r\rangle, w\rangle)$ is not in $L^{\prime \prime}$, and thus $(x, y) \notin L^{\prime \prime \prime}$.

If $(x, y) \in L$ then with probability $1 / 2 l_{n}$ we have $m=m^{*}$ as $m \leq \log 4 l_{n} d \leq$ $2 l_{n}$. In case $m=m^{*}$ there is exactly one witness $w$ for $(\langle x, r\rangle,\langle y, r\rangle)$ showing $(x, y) \in L^{\prime \prime \prime}$. The size of $A$ is at least $l_{n} d^{2}$, the size of $F^{2}$ is at most $16 l_{n}^{2} d^{2}$. If we choose $(a, b)$ at random in $F^{2}$ we have a $1 / 16 l_{n}$ chance of being in $A$. Thus, for fixed input $(x, y)$ the probability of choosing $r$ at random such that $m=m^{*}$ and $(a, b) \in A$ is at least $1 / 32 l_{n}^{2}$.

The class $\oplus \cdot \mathcal{C}$ is closed under majority reductions by Fact 2.12 . Thus, probability amplification is possible, and we get $L \in \mathrm{BP} \cdot \oplus \cdot \mathcal{C}$.

Theorem 2.16. (Toda) $\mathbf{P} H^{c c} \subseteq \mathrm{BP} \cdot \oplus \cdot \mathbf{P}^{c c}$.

Proof. We prove $\Sigma_{k}^{c c} \subseteq \mathrm{BP} \cdot \oplus \cdot \mathbf{P}^{c c}$ by induction on $k$ :

Case $k=0$ : The class $\mathbf{P}^{c c}$ is closed under many-one reductions. The class $\oplus \cdot \mathbf{P}^{c c}$ is also closed under many-one reductions by Fact 2.12 , because $\mathbf{P}^{c c}$ is closed under $\mathbf{P}^{c c}$-intersection, $\mathbf{P}^{c c}$-union, and conjunctive reductions. Thus, $\Sigma_{0}^{c c}=\mathbf{P}^{c c} \subseteq \oplus \cdot \mathbf{P}^{c c} \subseteq \mathrm{BP} \cdot \oplus \cdot \mathbf{P}^{c c}$ by the inclusion property of the $\oplus$ - and BP-operator (Observation 2.4).

Case $k \rightarrow k+1$ : It holds

$$
\begin{aligned}
\Sigma_{k+1}^{c c} & =\exists \cdot \mathrm{co} \cdot \Sigma_{k}^{c c} \\
& \subseteq \exists \cdot \mathrm{co} \cdot \mathrm{BP} \cdot \oplus \cdot \mathbf{P}^{c c} \\
& =\exists \cdot \mathrm{BP} \cdot \mathrm{co} \cdot \oplus \cdot \mathbf{P}^{c c} \\
& =\exists \cdot \mathrm{BP} \cdot \oplus \cdot \mathbf{P}^{c c} \\
& \subseteq \mathrm{BP} \cdot \oplus \cdot \mathrm{BP} \cdot \oplus \cdot \mathbf{P}^{c c} \\
& \subseteq \mathrm{BP} \cdot \mathrm{BP} \cdot \oplus \cdot \oplus \cdot \mathbf{P}^{c c} \\
& =\mathrm{BP} \cdot \mathrm{BP} \cdot \oplus \cdot \mathbf{P}^{c c} \\
& =\mathrm{BP} \cdot \oplus \cdot \mathbf{P}^{c c}
\end{aligned}
$$


(2) By Definition 2.2.

(3) By the induction hypothesis for $\Sigma_{k}^{c c}$ and monotonicity (Observation 2.5) of the operators co and $\exists$.

(4) By Observation 2.8.

(5) By closure under complement of $\oplus \cdot \mathbf{P}^{c c}$ (Observation 2.10).

(6) By the Valiant-Vazirani-Lemma (Lemma 2.15). Its application is possible, because $\mathrm{BP} \cdot \oplus \cdot \mathbf{P}^{c c}$ is closed under conjunctive reductions and $\mathbf{P}^{c c}$ intersection.

(7) By the Swapping-Lemma (Lemma 2.14) and monotonicity of the BPoperator (Observation 2.5). The Swapping-Lemma can be applied, because $\oplus \cdot \mathbf{P}^{c c}$ is closed under majority reductions.

(8) By idempotency of the $\oplus$-operator (Observation 2.6).

(9) By idempotency of the BP-operator (Observation 2.7). This holds because $\oplus \cdot \mathbf{P}^{c c}$ is closed under majority reductions.

Fact 2.17. $\mathbf{P}^{c c}\left(\mathbf{P P}^{c c}\right)=\mathbf{P}^{c c}\left(\# \mathbf{P}^{c c}\right)$.

Proof. Alice and Bob can compute every $\# \mathbf{P}^{c c}$-function $f$ by binary search with polylog communication asking oracle queries to $\operatorname{Graph}_{\leq}(f):=\{(\langle x, v\rangle,\langle y, v\rangle) \mid$ $\left.(v)_{2} \leq f(x, y)\right\} \in \mathbf{P P}^{c c}$.

Theorem 2.18. (Toda) $\mathbf{P} \mathbf{H}^{c c} \subseteq \mathrm{BP} \cdot \oplus \mathbf{P}^{c c} \subseteq \mathbf{P}^{c c}\left(\# \mathbf{P}^{c c}\right)=\mathbf{P}^{c c}\left(\mathbf{P} \mathbf{P}^{c c}\right)$.

Proof. Let $\operatorname{acc}_{\Pi}(x, y)$ denote the number of accepting paths of a nondeterministic protocol $\Pi$ on input $(x, y)$. The class $\# \mathbf{P}^{c c}$ contains all constant functions and is closed under addition and multiplication. If $\left(\Pi_{n}\right)_{n \in \mathbb{N}}$ is an efficient nondeterministic protocol family with $\operatorname{acc}_{\Pi}:=\left(\operatorname{acc}_{\Pi_{n}}\right)_{n \in \mathbb{N}}$ in $\# \mathbf{P}^{c c}$, and if we choose $p \in$ poly, then there exists an efficient nondeterministic protocol family $\left(\Pi_{n}^{\prime}\right)_{n \in \mathbb{N}}$ such that $\operatorname{acc}_{\Pi_{n}^{\prime}}(x, y)=\left(1+\operatorname{acc}_{\Pi_{n}}(x, y)^{\lceil p(\log n)\rceil}\right)^{\lceil p(\log n)\rceil}$. This proves $\mathrm{BP} \cdot \oplus \mathbf{P}^{c c} \subseteq \mathbf{P}^{c c}\left(\# \mathbf{P}^{c c}\right)$ as in the time complexity setting.

Let IP denote the inner product function (see [11, Ex. 1.25, p.12]), and let MAJ denote the majority function (see e.g. [9]). The corollary below considers the consequences of the unlikely case that the inner product or majority function can be computed with a constant number of alternations.

Corollary 2.19. It holds:
1. IP $\in \mathbf{P} \mathbf{H}^{c c}$ iff $\mathbf{P} \mathbf{H}^{c c}=\mathrm{BP} \cdot \oplus \mathbf{P}^{c c}$.
2. If $\mathbf{P} \mathbf{H}^{c c}=\mathbf{P S P A C E} \mathbf{E}^{c c}$ then $\mathbf{P} \mathbf{H}^{c c}=\mathrm{BP} \cdot \oplus \mathbf{P}^{c c}$.
3. $\mathrm{MAJ} \in \mathbf{P} \mathbf{H}^{c c}$ iff $\mathbf{P} \mathbf{H}^{c c}=\mathrm{BP} \cdot \oplus \mathbf{P}^{c c}=\mathbf{B P}^{c c}\left(\mathbf{P} \mathbf{P}^{c c}\right)$.
4. IP $\in \mathbf{P} \mathbf{H}^{c c}$ iff $\mathrm{MAJ} \in \mathbf{P} \mathbf{H}^{c c}$. 
Proof. 1. $\Rightarrow:$ IP $\in \mathbf{P} \mathbf{H}^{c c}$ implies $\oplus \mathbf{P}^{c c} \subseteq \mathbf{P H}^{c c}$ because IP is complete for $\oplus \mathbf{P}^{c c}$ under many-one reductions. Applying the BP-operator yields $\mathrm{BP} \cdot \oplus \mathbf{P}^{c c} \subseteq$ $\mathrm{BP} \cdot \mathbf{P} \mathbf{H}^{c c}=\mathbf{P} \mathbf{H}^{c c} \cdot \Leftarrow: \mathrm{IP} \in \oplus \mathbf{P}^{c c} \subseteq \mathrm{BP} \cdot \oplus \mathbf{P}^{c c}=\mathbf{P} \mathbf{H}^{c c}$.

2. Follows from $\oplus \mathbf{P}^{c c} \subseteq \mathbf{P S P A C E} \mathbf{E}^{c c}$ and (1.).

3. $\Rightarrow:$ MAJ $\in \mathbf{P H}^{c c}$ implies $\mathbf{P} \mathbf{P}^{c c} \subseteq \mathbf{P H}^{c c}$ because MAJ is complete for $\mathbf{P} \mathbf{P}^{c c}$ under many-one reductions. We obtain $\mathbf{B P} \mathbf{P P}^{c c}\left(\mathbf{P P}^{c c}\right) \subseteq \mathbf{B} \mathbf{P} \mathbf{P}^{c c}\left(\mathbf{P H}^{c c}\right)=$ $\mathbf{P} \mathbf{H}^{c c} \subseteq \mathrm{BP} \cdot \oplus \mathbf{P}^{c c} \subseteq \mathbf{P}^{c c}\left(\mathbf{P P}^{c c}\right) \subseteq \mathbf{B P P}^{c c}\left(\mathbf{P P}^{c c}\right) . \Leftarrow: \mathrm{MAJ} \in \mathbf{P P}^{c c} \subseteq$ $\operatorname{BPP}\left(\mathbf{P P}^{c c}\right)=\mathbf{P} \mathbf{H}^{c c}$.

4. Follows from (1.) and (3.).

\section{An alternating protocol for IP}

The class PSPACE ${ }^{c c}$ was defined as the class of languages which can be recognized with protocols using $(\log n)^{\mathcal{O}(1)}$ communication and $\mathcal{O}(\log n)$ alternations. In this section we show that languages in the class $\mathrm{BP} \cdot \oplus \mathbf{P}^{c c}$ can be recognized by alternating protocols using only $\mathcal{O}(\log n / \log \log n)$ many alternations. It is enough to give such a protocol for the inner product function, because IP is complete for $\oplus \mathbf{P}^{c c}$, and Schöning's generalization BP $\mathcal{C} \subseteq \exists \cdot \forall \cdot \mathcal{C} \cap \forall \cdot \exists \cdot \mathcal{C}$ of the well known result of Lautemann, which is easily transferred into the communication complexity context. For a proof, see [10, Prop. 2.24, p.76]. Fix an odd natural number $k$. Alice has input $x=x_{0} \ldots x_{n-1}$ and Bob has input $y=y_{0} \ldots y_{n-1}$. They execute the following alternating protocol $I_{k}(s, t, b)$ on their inputs:

If $(k \geq t-s+1)$ then Alice and Bob determine if $\operatorname{IP}_{t-s+1}\left(x_{s} \ldots x_{t}, y_{s} \ldots y_{t}\right)=$ $b$ using the trivial protocol (Alice sends her input; both compute the value by themselves). They return the value of the trivial protocol.

else Alice guesses the following strings and sends them to Bob:

1. $\exists S \subseteq\{0, \ldots, k-1\},|S|$ odd: (branch disjunctively)

2. $\exists \tilde{b} \in\{0,1\}:$ (branch disjunctively)

3. $\forall i \in S:$ (branch conjunctively)

4. $\forall j \in \bar{S}$ : (branch conjunctively)

5. $\forall h \in\{i, j\}:$ (branch conjunctively)

return $I_{k}\left(s_{1}, t_{1}, b_{1}\right)$, where $d:=t-s+1, B:=\left\lceil\frac{d}{k}\right\rceil, s_{1}:=h \cdot B, t_{1}:=$ $\min \{n-1,(h+1) \cdot B-1\}$, and if $(h=i)$ then $b_{1}:=b$ else $b_{1}:=\tilde{b}$.

Correctness. Divide each input $x$ and $y$ in an odd number $k$ of blocks of approximately equal sizes, i.e. $x=x^{(1)} \cdots x^{(k)}, y=y^{(1)} \cdots y^{(k)}$. It holds $\operatorname{IP}(x, y)=\sum_{i \in[k]} \operatorname{IP}\left(x^{(i)}, y^{(i)}\right) \bmod 2$. If $\operatorname{IP}(x, y)$ evaluates to 1 , there exists an odd number of blocks $S^{\prime} \subseteq[k]$ where IP evaluates to 1 and the values of IP cancel on $\overline{S^{\prime}}$. There are three cases:

1. $\operatorname{IP}\left(x^{(j)}, y^{(j)}\right)=0$ for all $j \in \overline{S^{\prime}}$. We set $S:=S^{\prime}$ and $\tilde{b}:=0$.

2. $\operatorname{IP}\left(x^{(j)}, y^{(j)}\right)=1$ for all $j \in \overline{S^{\prime}}$. We set $S:=S^{\prime}$ and $\tilde{b}:=1$.

3. There exist $j_{0}, j_{1} \in \bar{S}$ such that $\operatorname{IP}\left(x^{\left(j_{0}\right)}, y^{\left(j_{0}\right)}\right)=0$ and $\operatorname{IP}\left(x^{\left(j_{1}\right)}, y^{\left(j_{1}\right)}\right)=1$. The number of $j \in \overline{S^{\prime}}$ with $\operatorname{IP}\left(x^{(j)}, y^{(j)}\right)=1$ has to be even. We set $S:=S^{\prime} \cup\left\{j \in \overline{S^{\prime}} \mid \operatorname{IP}\left(x^{(j)}, y^{(j)}\right)=1\right\}$ and $\tilde{b}:=0$. Note that $|S|$ is odd. 
In all three cases we have obtained a set $S \subseteq[k]$ of odd cardinality and a $\tilde{b}$ such that $\operatorname{IP}\left(x^{(i)}, y^{(i)}\right)=1$ for all $i \in S$ and $\operatorname{IP}\left(x^{(j)}, y^{(j)}\right)=\tilde{b}$ for all $j \in \bar{S}$. The case when $\operatorname{IP}(x, y)$ evaluates to 0 is analogous. Thus, the protocol $I_{k}(s, t, b)$ accepts iff $\operatorname{IP}_{t-s+1}\left(x_{s} \ldots x_{t}, y_{s} \ldots y_{t}\right)=b$. The protocol $I_{k}(0, n-1,1)$ computes $\operatorname{IP}_{n}(x, y)$.

Communication costs. There are two alternations in each round and the number of rounds is bounded by $t=\log _{2} n / \log _{2} k$. If we choose an odd $k$ of size $(\log n)^{c}$ then the communication costs in each round are $\mathcal{O}(k)$ bits and the number of alternations is $\mathcal{O}(\log n / \log \log n)$. If $\operatorname{AComm}_{\mathcal{A}}(\mathcal{F})$ denotes the class of languages which can be recognized by alternating protocols using communication bounded by a function in $\mathcal{F}$ and a number of alternations bounded by a function in $\mathcal{A}$, we have obtained

Theorem 3.1. $\mathrm{BP} \cdot \oplus \mathbf{P}^{c c} \subseteq \mathbf{A C o m m}_{\mathcal{O}(\log n / \log \log n)}\left((\log n)^{\mathcal{O}(1)}\right)$.

\section{Acknowledgement}

I would like to express my deep gratitude to Martin Dietzfelbinger, Uwe Schöning and Jacobo Torán for careful reading, fruitful discussions, and their support.

\section{References}

[1] L. Babai, P. Frankl, and J. Simon. Complexity classes in communication complexity theory (preliminary version). In 27th Annual Symposium on Foundations of Computer Science, 27-29 October 1986, Toronto, Ontario, Canada, pages 337-347, 1986.

[2] J. L. Balcázar, J. Díaz, and J. Gabarró. Structural Complexity II. Texts in Theoretical Computer Science, An EATCS Series. Springer-Verlag, 1st edition, 1990.

[3] J. L. Balcázar, J. Díaz, and J. Gabarró. Structural Complexity I. Texts in Theoretical Computer Science, An EATCS Series. Springer-Verlag, 2nd edition, 1995.

[4] H. Buhrman, N. K. Vereshchagin, and R. de Wolf. On computation and communication with small bias. In 22nd Annual IEEE Conference on Computational Complexity (CCC 2007), 13-16 June 2007, San Diego, California, USA, pages 24-32. IEEE Computer Society, 2007.

[5] C. Damm, M. Krause, C. Meinel, and S. Waack. On relations between counting communication complexity classes. J. Comput. Syst. Sci., 69(2):259-280, 2004.

[6] D.-Z. Du and K.-I. Ko. Theory of Computational Complexity. Series in Discrete Mathematics and Optimization. Wiley-Interscience, 1st edition, 2000.

[7] L. Fortnow. Counting complexity. In Selman and Hemaspaandra [19], pages $81-107$.

[8] B. Halstenberg and R. Reischuk. Relations between communication complexity classes. J. Comput. Syst. Sci., 41(3):402-429, 1990. 
[9] H. Klauck. Rectangle size bounds and threshold covers in communication complexity. In 18th Annual IEEE Conference on Computational Complexity, 7-10 July 2003, Aarhus, Denmark, pages 118-134. IEEE Computer Society.

[10] J. Köbler, U. Schöning, and J. Torán. The Graph Isomorphism Problem Its Structural Complexity. Birkhäuser Boston, 1993.

[11] E. Kushilevitz and N. Nisan. Communication Complexity. Cambridge University Press, 1997.

[12] C. H. Papadimitriou and S. Zachos. Two remarks on the power of counting. In A. B. Cremers and H.-P. Kriegel, editors, Theoretical Computer Science, 6th GI-Conference, Dortmund, Germany, January 5-7, 1983, Proceedings, volume 145 of Lecture Notes in Computer Science, pages 269-276. SpringerVerlag, 1983.

[13] A. Razborov and A. Sherstov. The sign-rank of $\mathbf{A C}^{0}$. FOCS'08, to appear.

[14] U. Schöning. Complexity and Structure, volume 211 of Lecture Notes in Computer Science. Springer-Verlag, 1986.

[15] U. Schöning. The power of counting. In Selman [18], pages 204-223.

[16] U. Schöning. Probabilistic complexity classes and lowness. J. Comput. Syst. Sci., 39(1):84-100, 1989.

[17] U. Schöning. Recent highlights in structural complexity theory (invited talk). In SOFSEM'91, Nizké Tratry (CSFR), Conference Proceedings, pages 205-216. Springer-Verlag, December 1991.

[18] A. L. Selman, editor. Complexity Theory Retrospective, Foundations of Computing. Springer-Verlag, 1988.

[19] A. L. Selman and L. A. Hemaspaandra, editors. Complexity Theory Retrospective II, Foundations of Computing. Springer-Verlag, 1997.

[20] S. Toda. PP is as hard as the polynomial-time hierarchy. SIAM J. Comput., 20(5):865-877, 1991.

[21] L. G. Valiant and V. V. Vazirani. NP is as easy as detecting unique solutions. Theor. Comput. Sci., 47(3):85-93, 1986.

[22] A. C.-C. Yao. Some complexity questions related to distributive computing (preliminary report). In Conference Record of the Eleventh Annual ACM Symposium on Theory of Computing, 30 April-2 May, 1979, Atlanta, Georgia, USA, pages 209-213. ACM, 1979. 


\section{Liste der bisher erschienenen Ulmer Informatik-Berichte}

Einige davon sind per FTP von ftp.informatik.uni-ulm. de erhältlich

Die mit * markierten Berichte sind vergriffen

List of technical reports published by the University of Ulm

Some of them are available by FTP from ftp. informatik. uni-ulm. de

Reports marked with * are out of print

91-01 Ker-I Ko, P. Orponen, U. Schöning, O. Watanabe

Instance Complexity

91-02* K. Gladitz, H. Fassbender, H. Vogler

Compiler-Based Implementation of Syntax-Directed Functional Programming

91-03* Alfons Geser

Relative Termination

91-04* J. Köbler, U. Schöning, J. Toran

Graph Isomorphism is low for PP

91-05 Johannes Köbler, Thomas Thierauf

Complexity Restricted Advice Functions

91-06* Uwe Schöning

Recent Highlights in Structural Complexity Theory

91-07* F. Green, J. Köbler, J. Toran

The Power of Middle Bit

91-08* V.Arvind, Y. Han, L. Hamachandra, J. Köbler, A. Lozano, M. Mundhenk, A. Ogiwara, U. Schöning, R. Silvestri, T. Thierauf

Reductions for Sets of Low Information Content

92-01* Vikraman Arvind, Johannes Köbler, Martin Mundhenk

On Bounded Truth-Table and Conjunctive Reductions to Sparse and Tally Sets

92-02* Thomas Noll, Heiko Vogler

Top-down Parsing with Simulataneous Evaluation of Noncircular Attribute Grammars

92-03 Fakultät für Informatik

17. Workshop über Komplexitätstheorie, effiziente Algorithmen und Datenstrukturen

92-04* V. Arvind, J. Köbler, M. Mundhenk

Lowness and the Complexity of Sparse and Tally Descriptions

92-05* Johannes Köbler

Locating P/poly Optimally in the Extended Low Hierarchy

92-06* Armin Kühnemann, Heiko Vogler

Synthesized and inherited functions -a new computational model for syntax-directed semantics

92-07* Heinz Fassbender, Heiko Vogler

A Universal Unification Algorithm Based on Unification-Driven Leftmost Outermost Narrowing 
92-08* Uwe Schöning

On Random Reductions from Sparse Sets to Tally Sets

92-09* Hermann von Hasseln, Laura Martignon

Consistency in Stochastic Network

92-10 Michael Schmitt

A Slightly Improved Upper Bound on the Size of Weights Sufficient to Represent Any Linearly Separable Boolean Function

92-11 Johannes Köbler, Seinosuke Toda

On the Power of Generalized MOD-Classes

92-12 V. Arvind, J. Köbler, M. Mundhenk

Reliable Reductions, High Sets and Low Sets

92-13 Alfons Geser

On a monotonic semantic path ordering

92-14* Joost Engelfriet, Heiko Vogler

The Translation Power of Top-Down Tree-To-Graph Transducers

93-01 Alfred Lupper, Konrad Froitzheim

AppleTalk Link Access Protocol basierend auf dem Abstract Personal

Communications Manager

93-02 M.H. Scholl, C. Laasch, C. Rich, H.-J. Schek, M. Tresch

The COCOON Object Model

93-03 Thomas Thierauf, Seinosuke Toda, Osamu Watanabe

On Sets Bounded Truth-Table Reducible to P-selective Sets

93-04 Jin-Yi Cai, Frederic Green, Thomas Thierauf

On the Correlation of Symmetric Functions

93-05 K.Kuhn, M.Reichert, M. Nathe, T. Beuter, C. Heinlein, P. Dadam

A Conceptual Approach to an Open Hospital Information System

93-06 Klaus Gaßner

Rechnerunterstützung für die konzeptuelle Modellierung

93-07 Ullrich Keßler, Peter Dadam

Towards Customizable, Flexible Storage Structures for Complex Objects

94-01 Michael Schmitt

On the Complexity of Consistency Problems for Neurons with Binary Weights

94-02 Armin Kühnemann, Heiko Vogler

A Pumping Lemma for Output Languages of Attributed Tree Transducers

94-03 Harry Buhrman, Jim Kadin, Thomas Thierauf

On Functions Computable with Nonadaptive Queries to NP

94-04 Heinz Faßbender, Heiko Vogler, Andrea Wedel

Implementation of a Deterministic Partial E-Unification Algorithm for Macro Tree

Transducers 
94-05 V. Arvind, J. Köbler, R. Schuler

On Helping and Interactive Proof Systems

94-06 Christian Kalus, Peter Dadam

Incorporating record subtyping into a relational data model

94-07 Markus Tresch, Marc H. Scholl

A Classification of Multi-Database Languages

94-08 Friedrich von Henke, Harald Rueß

Arbeitstreffen Typtheorie: Zusammenfassung der Beiträge

94-09 F.W. von Henke, A. Dold, H. Rueß, D. Schwier, M. Strecker

Construction and Deduction Methods for the Formal Development of Software

94-10 Axel Dold

Formalisierung schematischer Algorithmen

94-11 Johannes Köbler, Osamu Watanabe

New Collapse Consequences of NP Having Small Circuits

94-12 Rainer Schuler

On Average Polynomial Time

94-13 Rainer Schuler, Osamu Watanabe

Towards Average-Case Complexity Analysis of NP Optimization Problems

94-14 Wolfram Schulte, Ton Vullinghs

Linking Reactive Software to the X-Window System

94-15 Alfred Lupper

Namensverwaltung und Adressierung in Distributed Shared Memory-Systemen

94-16 Robert Regn

Verteilte Unix-Betriebssysteme

94-17 Helmuth Partsch

Again on Recognition and Parsing of Context-Free Grammars:

Two Exercises in Transformational Programming

94-18 Helmuth Partsch

Transformational Development of Data-Parallel Algorithms: an Example

95-01 Oleg Verbitsky

On the Largest Common Subgraph Problem

95-02 Uwe Schöning

Complexity of Presburger Arithmetic with Fixed Quantifier Dimension

95-03 Harry Buhrman,Thomas Thierauf

The Complexity of Generating and Checking Proofs of Membership

95-04 Rainer Schuler, Tomoyuki Yamakami

Structural Average Case Complexity

95-05 Klaus Achatz, Wolfram Schulte

Architecture Indepentent Massive Parallelization of Divide-And-Conquer Algorithms 
95-06 Christoph Karg, Rainer Schuler

Structure in Average Case Complexity

95-07 P. Dadam, K. Kuhn, M. Reichert, T. Beuter, M. Nathe

ADEPT: Ein integrierender Ansatz zur Entwicklung flexibler, zuverlässiger kooperierender Assistenzsysteme in klinischen Anwendungsumgebungen

95-08 Jürgen Kehrer, Peter Schulthess

Aufbereitung von gescannten Röntgenbildern zur filmlosen Diagnostik

95-09 Hans-Jörg Burtschick, Wolfgang Lindner

On Sets Turing Reducible to P-Selective Sets

95-10 Boris Hartmann

Berücksichtigung lokaler Randbedingung bei globaler Zieloptimierung mit neuronalen Netzen am Beispiel Truck Backer-Upper

95-12 Klaus Achatz, Wolfram Schulte

Massive Parallelization of Divide-and-Conquer Algorithms over Powerlists

95-13 Andrea Mößle, Heiko Vogler

Efficient Call-by-value Evaluation Strategy of Primitive Recursive Program Schemes

95-14 Axel Dold, Friedrich W. von Henke, Holger Pfeifer, Harald Rueß

A Generic Specification for Verifying Peephole Optimizations

96-01 Ercüment Canver, Jan-Tecker Gayen, Adam Moik

Formale Entwicklung der Steuerungssoftware für eine elektrisch ortsbediente Weiche mit VSE

96-02 Bernhard Nebel

Solving Hard Qualitative Temporal Reasoning Problems: Evaluating the Efficiency of Using the ORD-Horn Class

96-03 Ton Vullinghs, Wolfram Schulte, Thilo Schwinn

An Introduction to TkGofer

96-04 Thomas Beuter, Peter Dadam

Anwendungsspezifische Anforderungen an Workflow-Mangement-Systeme am

Beispiel der Domäne Concurrent-Engineering

96-05 Gerhard Schellhorn, Wolfgang Ahrendt

Verification of a Prolog Compiler - First Steps with KIV

96-06 Manindra Agrawal, Thomas Thierauf

Satisfiability Problems

96-07 Vikraman Arvind, Jacobo Torán

A nonadaptive NC Checker for Permutation Group Intersection

96-08 David Cyrluk, Oliver Möller, Harald Rueß

An Efficient Decision Procedure for a Theory of Fix-Sized Bitvectors with Composition and Extraction

96-09 Bernd Biechele, Dietmar Ernst, Frank Houdek, Joachim Schmid, Wolfram Schulte Erfahrungen bei der Modellierung eingebetteter Systeme mit verschiedenen SA/RTAnsätzen 
96-10 Falk Bartels, Axel Dold, Friedrich W. von Henke, Holger Pfeifer, Harald Rueß Formalizing Fixed-Point Theory in PVS

96-11 Axel Dold, Friedrich W. von Henke, Holger Pfeifer, Harald Rueß Mechanized Semantics of Simple Imperative Programming Constructs

96-12 Axel Dold, Friedrich W. von Henke, Holger Pfeifer, Harald Rueß Generic Compilation Schemes for Simple Programming Constructs

96-13 Klaus Achatz, Helmuth Partsch From Descriptive Specifications to Operational ones: A Powerful Transformation Rule, its Applications and Variants

97-01 Jochen Messner

Pattern Matching in Trace Monoids

97-02 Wolfgang Lindner, Rainer Schuler

A Small Span Theorem within P

97-03 Thomas Bauer, Peter Dadam

A Distributed Execution Environment for Large-Scale Workflow Management Systems with Subnets and Server Migration

97-04 Christian Heinlein, Peter Dadam

Interaction Expressions - A Powerful Formalism for Describing Inter-Workflow Dependencies

97-05 Vikraman Arvind, Johannes Köbler

On Pseudorandomness and Resource-Bounded Measure

97-06 Gerhard Partsch

Punkt-zu-Punkt- und Mehrpunkt-basierende LAN-Integrationsstrategien für den digitalen Mobilfunkstandard DECT

97-07 Manfred Reichert, Peter Dadam

$A D E P T_{\text {flex }}$ - Supporting Dynamic Changes of Workflows Without Loosing Control

97-08 Hans Braxmeier, Dietmar Ernst, Andrea Mößle, Heiko Vogler

The Project NoName - A functional programming language with its development environment

97-09 Christian Heinlein

Grundlagen von Interaktionsausdrücken

97-10 Christian Heinlein

Graphische Repräsentation von Interaktionsausdrücken

97-11 Christian Heinlein

Sprachtheoretische Semantik von Interaktionsausdrücken

97-12 Gerhard Schellhorn, Wolfgang Reif

Proving Properties of Finite Enumerations: A Problem Set for Automated Theorem Provers 
97-13 Dietmar Ernst, Frank Houdek, Wolfram Schulte, Thilo Schwinn

Experimenteller Vergleich statischer und dynamischer Softwareprüfung für eingebettete Systeme

97-14 Wolfgang Reif, Gerhard Schellhorn

Theorem Proving in Large Theories

97-15 Thomas Wennekers

Asymptotik rekurrenter neuronaler Netze mit zufälligen Kopplungen

97-16 Peter Dadam, Klaus Kuhn, Manfred Reichert

Clinical Workflows - The Killer Application for Process-oriented Information Systems?

97-17 Mohammad Ali Livani, Jörg Kaiser

EDF Consensus on CAN Bus Access in Dynamic Real-Time Applications

97-18 Johannes Köbler,Rainer Schuler

Using Efficient Average-Case Algorithms to Collapse Worst-Case Complexity Classes

98-01 Daniela Damm, Lutz Claes, Friedrich W. von Henke, Alexander Seitz, Adelinde

Uhrmacher, Steffen Wolf

Ein fallbasiertes System für die Interpretation von Literatur zur Knochenheilung

98-02 Thomas Bauer, Peter Dadam

Architekturen für skalierbare Workflow-Management-Systeme - Klassifikation und Analyse

98-03 Marko Luther, Martin Strecker

A guided tour through Typelab

98-04 Heiko Neumann, Luiz Pessoa

Visual Filling-in and Surface Property Reconstruction

98-05 Ercüment Canver

Formal Verification of a Coordinated Atomic Action Based Design

98-06 Andreas Küchler

On the Correspondence between Neural Folding Architectures and Tree Automata

98-07 Heiko Neumann, Thorsten Hansen, Luiz Pessoa

Interaction of ON and OFF Pathways for Visual Contrast Measurement

98-08 Thomas Wennekers

Synfire Graphs: From Spike Patterns to Automata of Spiking Neurons

98-09 Thomas Bauer, Peter Dadam

Variable Migration von Workflows in $A D E P T$

98-10 Heiko Neumann, Wolfgang Sepp

Recurrent V1 - V2 Interaction in Early Visual Boundary Processing

98-11 Frank Houdek, Dietmar Ernst, Thilo Schwinn

Prüfen von C-Code und Statmate/Matlab-Spezifikationen: Ein Experiment 
98-12 Gerhard Schellhorn

Proving Properties of Directed Graphs: A Problem Set for Automated Theorem Provers

98-13 Gerhard Schellhorn, Wolfgang Reif

Theorems from Compiler Verification: A Problem Set for Automated Theorem Provers

98-14 Mohammad Ali Livani

SHARE: A Transparent Mechanism for Reliable Broadcast Delivery in CAN

98-15 Mohammad Ali Livani, Jörg Kaiser

Predictable Atomic Multicast in the Controller Area Network (CAN)

99-01 Susanne Boll, Wolfgang Klas, Utz Westermann

A Comparison of Multimedia Document Models Concerning Advanced Requirements

99-02 Thomas Bauer, Peter Dadam

Verteilungsmodelle für Workflow-Management-Systeme - Klassifikation und

Simulation

99-03 Uwe Schöning

On the Complexity of Constraint Satisfaction

99-04 Ercument Canver

Model-Checking zur Analyse von Message Sequence Charts über Statecharts

99-05 Johannes Köbler, Wolfgang Lindner, Rainer Schuler

Derandomizing RP if Boolean Circuits are not Learnable

99-06 Utz Westermann, Wolfgang Klas

Architecture of a DataBlade Module for the Integrated Management of Multimedia Assets

99-07 Peter Dadam, Manfred Reichert

Enterprise-wide and Cross-enterprise Workflow Management: Concepts, Systems, Applications. Paderborn, Germany, October 6, 1999, GI-Workshop Proceedings, Informatik' 99

99-08 Vikraman Arvind, Johannes Köbler

Graph Isomorphism is Low for $\mathrm{ZPP}^{\mathrm{NP}}$ and other Lowness results

99-09 Thomas Bauer, Peter Dadam

Efficient Distributed Workflow Management Based on Variable Server Assignments

2000-02 Thomas Bauer, Peter Dadam

Variable Serverzuordnungen und komplexe Bearbeiterzuordnungen im Workflow-

Management-System ADEPT

2000-03 Gregory Baratoff, Christian Toepfer, Heiko Neumann

Combined space-variant maps for optical flow based navigation

2000-04 Wolfgang Gehring

Ein Rahmenwerk zur Einführung von Leistungspunktsystemen 
2000-05 Susanne Boll, Christian Heinlein, Wolfgang Klas, Jochen Wandel

Intelligent Prefetching and Buffering for Interactive Streaming of MPEG Videos

2000-06 Wolfgang Reif, Gerhard Schellhorn, Andreas Thums

Fehlersuche in Formalen Spezifikationen

2000-07 Gerhard Schellhorn, Wolfgang Reif (eds.)

FM-Tools 2000: The $4^{\text {th }}$ Workshop on Tools for System Design and Verification

2000-08 Thomas Bauer, Manfred Reichert, Peter Dadam

Effiziente Durchführung von Prozessmigrationen in verteilten Workflow-

Management-Systemen

2000-09 Thomas Bauer, Peter Dadam

Vermeidung von Überlastsituationen durch Replikation von Workflow-Servern in ADEPT

2000-10 Thomas Bauer, Manfred Reichert, Peter Dadam

Adaptives und verteiltes Workflow-Management

2000-11 Christian Heinlein

Workflow and Process Synchronization with Interaction Expressions and Graphs

2001-01 Hubert Hug, Rainer Schuler

DNA-based parallel computation of simple arithmetic

2001-02 Friedhelm Schwenker, Hans A. Kestler, Günther Palm

3-D Visual Object Classification with Hierarchical Radial Basis Function Networks

2001-03 Hans A. Kestler, Friedhelm Schwenker, Günther Palm

RBF network classification of ECGs as a potential marker for sudden cardiac death

2001-04 Christian Dietrich, Friedhelm Schwenker, Klaus Riede, Günther Palm

Classification of Bioacoustic Time Series Utilizing Pulse Detection, Time and

Frequency Features and Data Fusion

2002-01 Stefanie Rinderle, Manfred Reichert, Peter Dadam

Effiziente Verträglichkeitsprüfung und automatische Migration von Workflow-

Instanzen bei der Evolution von Workflow-Schemata

2002-02 Walter Guttmann

Deriving an Applicative Heapsort Algorithm

2002-03 Axel Dold, Friedrich W. von Henke, Vincent Vialard, Wolfgang Goerigk

A Mechanically Verified Compiling Specification for a Realistic Compiler

2003-01 Manfred Reichert, Stefanie Rinderle, Peter Dadam

A Formal Framework for Workflow Type and Instance Changes Under Correctness Checks

2003-02 Stefanie Rinderle, Manfred Reichert, Peter Dadam

Supporting Workflow Schema Evolution By Efficient Compliance Checks

2003-03 Christian Heinlein

Safely Extending Procedure Types to Allow Nested Procedures as Values 
2003-04 Stefanie Rinderle, Manfred Reichert, Peter Dadam

On Dealing With Semantically Conflicting Business Process Changes.

2003-05 Christian Heinlein

Dynamic Class Methods in Java

2003-06 Christian Heinlein

Vertical, Horizontal, and Behavioural Extensibility of Software Systems

2003-07 Christian Heinlein

Safely Extending Procedure Types to Allow Nested Procedures as Values

(Corrected Version)

2003-08 Changling Liu, Jörg Kaiser

Survey of Mobile Ad Hoc Network Routing Protocols)

2004-01 Thom Frühwirth, Marc Meister (eds.)

First Workshop on Constraint Handling Rules

2004-02 Christian Heinlein

Concept and Implementation of $\mathrm{C}+++$, an Extension of $\mathrm{C}++$ to Support User-Defined

Operator Symbols and Control Structures

2004-03 Susanne Biundo, Thom Frühwirth, Günther Palm(eds.)

Poster Proceedings of the 27th Annual German Conference on Artificial Intelligence

2005-01 Armin Wolf, Thom Frühwirth, Marc Meister (eds.)

19th Workshop on (Constraint) Logic Programming

2005-02 Wolfgang Lindner (Hg.), Universität Ulm, Christopher Wolf (Hg.) KU Leuven

2. Krypto-Tag - Workshop über Kryptographie, Universität Ulm

2005-03 Walter Guttmann, Markus Maucher

Constrained Ordering

2006-01 Stefan Sarstedt

Model-Driven Development with ACTIVECHARTS, Tutorial

2006-02 Alexander Raschke, Ramin Tavakoli Kolagari

Ein experimenteller Vergleich zwischen einer plan-getriebenen und einer leichtgewichtigen Entwicklungsmethode zur Spezifikation von eingebetteten

Systemen

2006-03 Jens Kohlmeyer, Alexander Raschke, Ramin Tavakoli Kolagari

Eine qualitative Untersuchung zur Produktlinien-Integration über

Organisationsgrenzen hinweg

2006-04 Thorsten Liebig

Reasoning with OWL - System Support and Insights -

2008-01 H.A. Kestler, J. Messner, A. Müller, R. Schuler

On the complexity of intersecting multiple circles for graphical display 
2008-02 Manfred Reichert, Peter Dadam, Martin Jurisch,l Ulrich Kreher, Kevin Göser, Markus Lauer

Architectural Design of Flexible Process Management Technology

2008-03 Frank Raiser

Semi-Automatic Generation of CHR Solvers from Global Constraint Automata

2008-04 Ramin Tavakoli Kolagari, Alexander Raschke, Matthias Schneiderhan, Ian Alexander Entscheidungsdokumentation bei der Entwicklung innovativer Systeme für produktlinien-basierte Entwicklungsprozesse

2008-05 Markus Kalb, Claudia Dittrich, Peter Dadam

Support of Relationships Among Moving Objects on Networks

2008-06 Matthias Frank, Frank Kargl, Burkhard Stiller (Hg.)

WMAN 2008 - KuVS Fachgespräch über Mobile Ad-hoc Netzwerke

2008-07 M. Maucher, U. Schöning, H.A. Kestler

An empirical assessment of local and population based search methods with different degrees of pseudorandomness

2008-08 Henning Wunderlich

Covers have structure

2008-09 Karl-Heinz Niggl, Henning Wunderlich

Implicit characterization of FPTIME and NC revisited

2008-10 Henning Wunderlich

On span- $\mathrm{P}^{\mathrm{cc}}$ and related classes in structural communication complexity

2008-11 M. Maucher, U. Schöning, H.A. Kestler

On the different notions of pseudorandomness

2008-12 Henning Wunderlich

On Toda's Theorem in structural communication complexity 

Ulmer Informatik-Berichte

ISSN 0939-5091

Herausgeber:

Universität UIm

Fakultät für Ingenieurwissenschaften und Informatik 89069 Ulm 